\title{
Evaluation and Active Control of Clustered Hall Thruster Discharge Oscillations
}

\author{
Robert B. Lobbia $^{*}$ and Alec D. Gallimore. ${ }^{\dagger}$ \\ The University of Michigan, Ann Arbor, MI, 48109
}

\begin{abstract}
While preliminary experiments with a cluster of four Busek 200-Watt (BHT-200-X3) Closed Drift Hall thrusters indicate reasonable performance scalability from single thruster experiments, cluster plume interactions are believed to give rise to an increased ion energy spectrum as well as cross-talk and synchronization between the thruster discharges. Recent experiments with a higher power cluster of four 600-Watt (BHT-600) Hall thrusters have exhibited similar clustering behavior. Discharge voltage and current characteristics are studied in detail with frequency magnitude and phase analysis from low frequencies up to $100 \mathrm{kHz}$. Modulation of the electromagnetic circuit current provides data for the analysis of multiple correlations between the various signals, thereby quantifying all interdependencies. Accentuation and shifting of various modal features (including the so called "breathing" mode) are observed in the clustered configuration. We are then motivated to provide active stabilization of the observed oscillations in an effort to improve thruster lifetimes, overall efficiency, and performance. After identification of the thruster cluster system, various models and control algorithms are developed to provide stabilizing feedback control to the clustered Hall thrusters. Application of this control in real time has shown reductions in the discharge oscillations during thruster startup and steady operation.
\end{abstract}

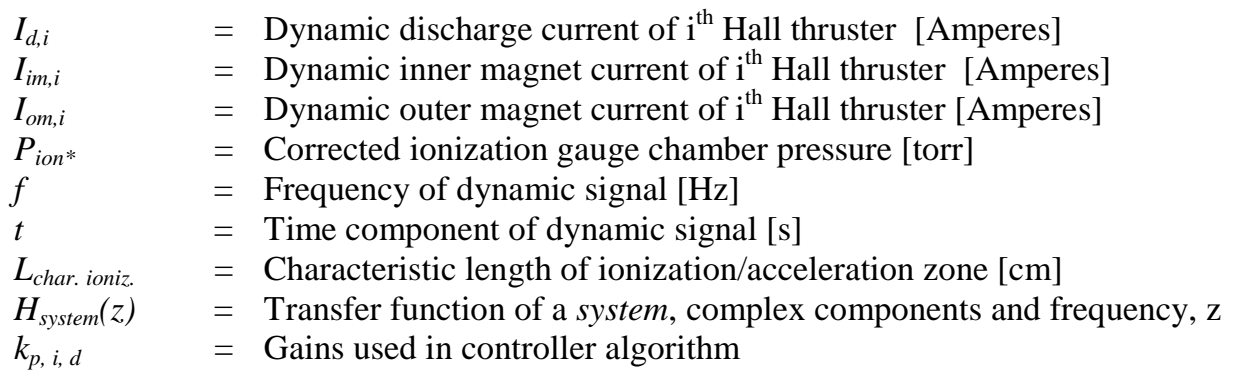

\section{Introduction}

\begin{abstract}
CLOSED drift Hall thrusters are used in many satellites today for station keeping and orbit boosting and an increasing number of spacecraft under development by the Air Force and NASA are utilizing this efficient means of propulsion ${ }^{1-3}$. Originally developed and extensively tested in the former Soviet Union, these thrusters employ orthogonal magnetic and electric fields to trap an ionizing layer of electrons and accelerate the generated ions, often achieving specific impulse performance in excess of 2000 seconds.
\end{abstract}

\section{A. Clustering of Hall Thrusters}

The enormous vacuum chamber pumping rates required to run higher power Hall thrusters motivated the use of clustered configurations to allow for the testing of smaller individual thrusters ${ }^{4}$. Although, a cluster of thrusters totaling a high power level of $100 \mathrm{~kW}$ will result in a lower thrust efficiency, a higher system dry mass and a shorter

\footnotetext{
${ }^{*}$ PhD Candidate, Aerospace Engineering Dept., FXB Building Room 1056, AIAA Student Member.

${ }^{\dagger}$ Professor, Aerospace Engineering Dept., Associate Fellow AIAA.
} 
lifetime than a single (monolithic) larger thruster of equal power, the advantages of the clustered approach include: $\mathrm{n}-1$ system redundancy, $\mathrm{n}$ step fully optimized throttlability, and cheaper system development ${ }^{5}$.

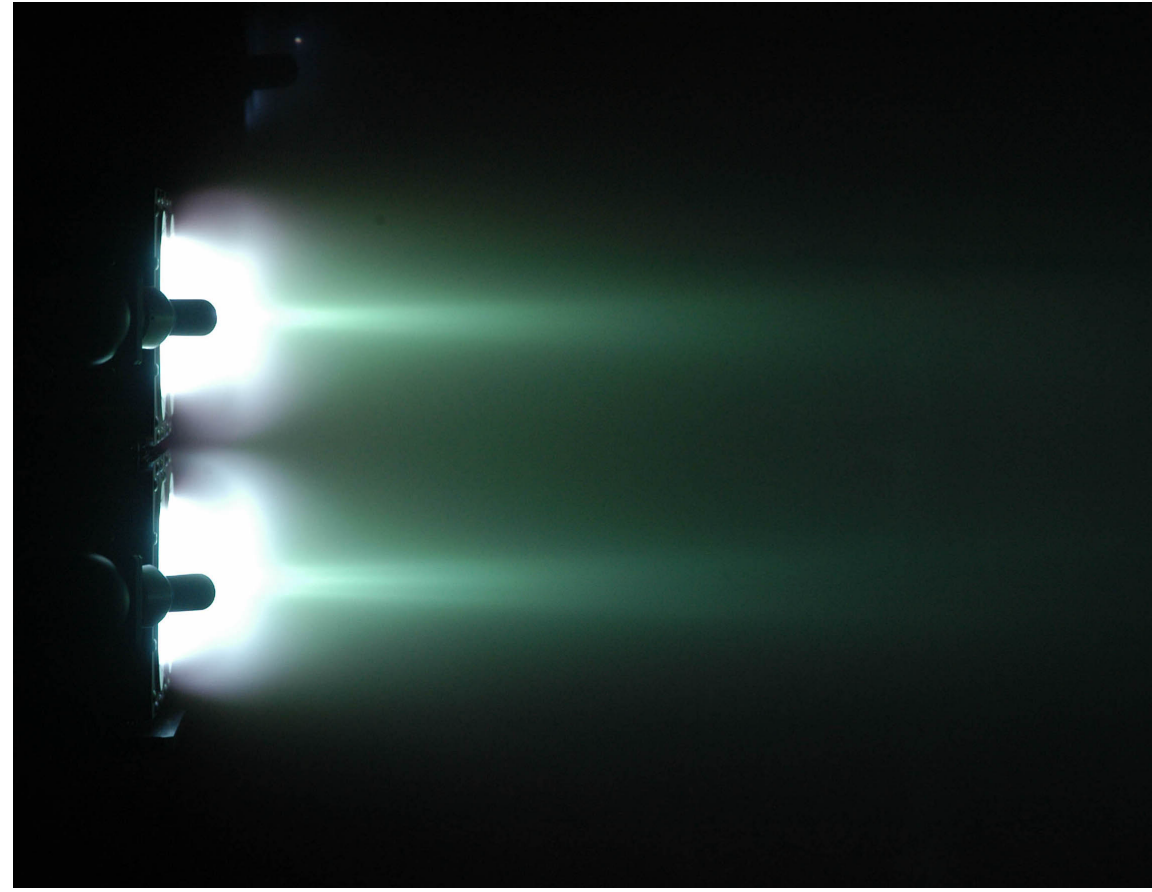

Figure 1. Cluster of four BHT-600 Hall Thrusters. Xenon discharge of $300 \mathrm{~V}$ and 2A per thruster in PEPL's LVTF (Large Vacuum Test Facility).

\section{B. Plasma Instabilities and Oscillatory Modes}

Inherent to the operation of all electric propulsion devices utilizing ionized particles are the plethora of plasma instabilities and oscillatory modes whose amplitudes can easily overwhelm the DC values of the operational discharge conditions. Self-interaction of plasma particles though the propagation of electromagnetic signals near the speed of light gives rise to a broad spectrum of plasma oscillatory modes. In a Hall thruster the electron plasma frequency is the most rapid of these plasma oscillations $(\sim 2 \mathrm{GHz})$, while the ioncyclotron frequency is the slowest $(\sim 1 \mathrm{kHz})^{10,12}$. Between these two frequencies there are at least seven additional oscillatory modes including the

well-studied breathing-mode, the rotating spike ionization instability, and the azimuthal gradient-induced instability. These instabilities were first observed in the operation of early plasma thrusters in the late 1960's and early 1970's by James, Lowder, Morozov, Tilinin, and others. Since then, recent work by Cappelli, Choueiri, and others has expanded upon these early results and developed theoretical models to further explain the origin of these instabilities. These findings have shown that the spectral response of the plasma depends on the position inside the thruster, ion mass and density, ion-accelerating discharge voltage, channel geometry, and the magnetic field gradient and magnitude. All of this prior thruster research in the realm of plasma-thruster instabilities has focused on the relationship of these modes to various discharge settings ${ }^{7-15}$ and thruster geometries ${ }^{16-17}$. While the use of active feedback control for mitigating these instabilities has been suggested in the past ${ }^{9,10}$, there has yet to be an actively controlled Hall thruster in closed loop at frequencies near the dominant breathing mode. The preliminary closed loop control results presented in this paper show a long overlooked means of dynamically stabilizing Hall thruster operation.

\section{Active Plasma Control Background}

Outside the field of plasma propulsion, plasma fusion researchers have been investigating and successfully employing feedback control of contained plasmas in tokamak, spheromak, mirror, and other devices since the early $1970 \mathrm{~s}^{18}$. The advent of modern computing and high-speed digital signal processing within the last 15 years has enabled the field and applications of feedback control to blossom. The general non-linear, chaotic, non-repeatable, and high frequency nature of plasmas had discouraged many researchers until the computational power and speed of modern computers made the problem less daunting. From a physical standpoint, it is clear that feedback control cannot be used to control every unstable mode since chaotic electromagnetic signals conduct across the plasma at rates nearly equivalent to the speed of light; nevertheless, the lower frequency modes $(<100 \mathrm{kHz})$ can be controlled with off-the-shelf DSPs, clever programming, and hardware (power supplies, sensors/actuators, filters, etc.) with minimal latencies (combined to less than $10 \mu \mathrm{s}$ for $100 \mathrm{kHz}$ closed loop control). Plasma fusion applications of feedback control have utilized various types of actuators and sensors including electrically biased plates, pulsed microwave and particle beams, and near-wall poloidal electromagnets ${ }^{19}$. Since Hall thrusters already utilize magnetic coils, the integration of feedback control into these thrusters does not require significant redesign. 
Although typical plasma properties associated with a Hall type plasma thruster differ substantially from that of a fusion type plasma, the tremendous success achieved with closed loop control $<10 \mathrm{kHz}$ in programs such as the DIIID tokamak ${ }^{19}$ implies that there exists an enormous untapped potential for increasing overall performance of stationary plasma thrusters, as well as other types of EP (Electric Propulsion) devices.

\section{Hall Thruster and Plasma Modeling}

Past numerical ${ }^{13,14}$ simulations as well as recent models ${ }^{20}$ of Hall thrusters are quite capable of reproducing a similar spectra of discharge current oscillations. Indeed, just as is the case with real Hall thrusters, some simulations have difficulty running in a stable manner due to the dominance of these numerically observed discharge oscillations during initial startup. The mere fact that a simulation can qualitatively reproduce the same oscillatory modes observed experimentally shows promise that controlling the physics of certain instabilities may not be as intractable as previously feared. While non-linear controllers are indeed available, the speed and relative simplicity of classical linear control theory is desirable as a starting point for the investigation of actively controlled Hall thrusters. A great deal of theoretical modeling relevant to the application of feedback control in fusion research devices is already developed ${ }^{21}$ and similar models can conceivably be developed for stationary plasma thrusters. In general, feedback control can be applied to any system without the prerequisite of a theoretical model; however, a model facilitates the development an optimized control scheme more readily and with better physical definition. Since existing computational Hall thruster models are numerically expensive and inaccurate, direct experimental input-output experiments are performed to create a state space or transfer function, $H_{\text {thruster }}$, empirical models based on the Hall thruster system.

\section{Motivation}

Although prior research ${ }^{5}$ with the 200W BHT-200-X3 Hall cluster has shown that most clustered plasma properties including ion and electron densities, are merely a linear superposition of the properties of a single thruster, the physical mechanisms of uniquely-clustered phenomena, such as current stealing, shared cathode operation, and various plume interactions, and have yet to be fully understood. A major focus of this article is placed upon the effect of clustering on the dynamic characteristics of the discharge current oscillations.

Closed loop feedback control is appropriate in the study of clustered (as well as single) thruster operation due to the abundance of input and output signals. This multitude of signals is fully exploited in the examination and quantification of crosstalk (cross-correlations) between the thrusters. Crosstalk, believed to occur primarily due to the thruster plumes interacting with one another, create a backwards (towards thruster) propagating disturbance affecting the instantaneous discharge current. Ultimately, it is expected that a stabilizing feedback controller will increase thruster lifetimes, improve efficiencies, and increase robustness to the thruster system. There may also exist modes of Hall thruster operation in which the discharge settings (including electromagnet currents) are intentionally oscillatory or pulsed (e.g. square wave excitation) in order to achieve higher thrust or more precise spacecraft trajectory correction maneuvers. Therefore, it is critical that a better understanding of Hall thruster dynamics, not just instabilities, is attained.

\section{Experimental Setup}

\section{E. Vacuum Chamber}

Experiments with the BHT-600 cluster used for this research were performed in the Plasmadynamics and Electric Propulsion Laboratory (PEPL) LVTF. This cylindrical, round-capped vacuum chamber measures $9 \mathrm{~m}$ end to end and $6 \mathrm{~m}$ in diameter. Four of the seven cryo pumps are utilized to achieve a pumping rate of $140,000 \mathrm{l} / \mathrm{s}$ on xenon. Two ionization gauges are used to measure the chamber pressure (corrected for xenon ${ }^{22}$ ). During single thruster operation, the corrected pressure was $P_{i o n} * 2.0 \times 10^{-6}$ torr. During the full, four thruster cluster operation, the correction chamber pressure increased to $P_{\text {ion }} * 4.5 \times 10^{-}$ ${ }^{6}$ torr.

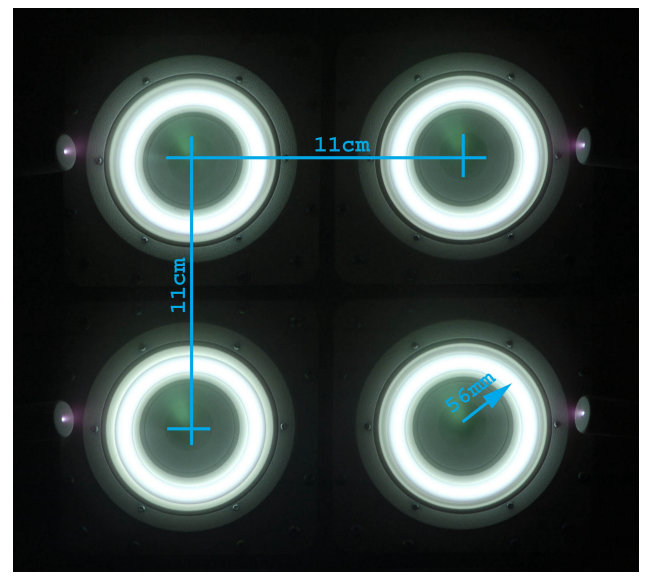

Figure 1. Hall thruster cluster dimensions and configuration. Photo taken through plume; hence bright spot to upper left of each discharge channel center. 


\section{F. Thruster Configuration and Specifications}

Each thruster uses its own cathode and its own set of power supplies so that there are independent and electrically isolated (from each other and chamber ground) discharge, heater, keeper, inner-magnet, and outermagnet regulated supplies. The cathode common potential is shared by the first three power supplies in the prior list for each thruster. While it is certainly feasible to run the entire cluster off a single set of shared supplies (giving rise to an interesting condition of current sharing), the premise of this paper is to isolate the plasma interactions, minimizing the effect of the electrical circuit on the operation of each individual thruster.

As seen in Figure 1, each Hall thruster has a discharge channel mean diameter of $5.6 \mathrm{~cm}$ and the thruster-tothruster centerline separation (horizontally and vertically) measures $11 \mathrm{~cm}$. The cluster is positioned in the approximate center of the LVTF with the plume impinging upon a series of graphite beam dump panels that cover the end of the chamber. The four cryo pumps are located the wall opposite the beam dump and the main ionization pressure gauge is located approximately inline with the cluster (both having nearly the same separation from the pumps). Prior to the acquisition of any data the Busek cluster cathodes go through a 5 hour conditioning procedure, through which most atmospheric contaminants (in particular, water and oxygen) are removed. The cluster is then run at $200 \mathrm{~V}$ and $2 \mathrm{~A}$ until a steady state operation (post startup transient ${ }^{6,7}$ ) is achieved at this point, the discharge voltage is increased to $300 \mathrm{~V}$ for the duration of the tests.

\section{G. Data Acquisition and Signal Control System}

The data acquisition (DAQ) system consists of a United Electronic Instruments AtoD (analog to digital) and DtoA card in a custom built Pentium 4 based PC. Each of the eight input AtoD channels are preceded by a sample and hold circuit to allow true simultaneous sampling of all channels. A Motorola $66 \mathrm{MHz} 56301$ onboard DSP allows for the simultaneous gap-free operation of the AtoD and DtoA subsystems at their full speed of $500 \mathrm{kHz}-$ even while running under Microsoft Windows XP. While basic input/output experiments (used to generate Bode plots) use a simple DOS program to acquire voltage signals, the closed loop feedback control experiments use the DAQ system in target mode. In this manner, a small operating system (OS) kernel ( $<2 \mathrm{MB})$ is loaded into the DAQ PC's memory to enable a minimization of OS latencies between the PC (processor, memory, hard-disk) and the UEI

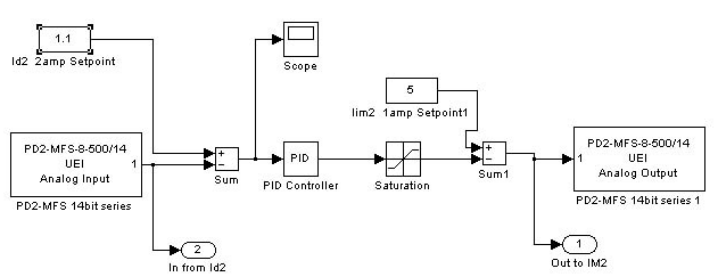

Figure 1. Example of a block diagram for feedback control. card. A host PC then sends a specially compiled Matlab Simulink block diagram to the target PC over a TCP/IP connection (see Figure 1). The target PC then executes the received code:

1. Gathering inputs from the AtoD channels

2. Processing the control algorithm

3. Updating the output control signal a stop command from the host. During each iteration of the control loop, input and output signals (as well as clock timing information) are recorded in the target PC's memory and later downloaded for post run analysis. It should be noted that currents (discharge and electromagnet) were measured using high speed (>100kHz) magneto-resistive current sensors as opposed to isolated shunts (which have frequency responses $<20 \mathrm{kHz}$ due to the lower bandwidth the isolation amplifiers).

\section{Experimental Results}

\section{H. Time Domain Response}

An example of the raw time-domain signals from a given input/output experiment is given in Figure 2. The dominant feature is the wide band large amplitude of the discharge current oscillations (shown in solid blue). This specific instability is believed to be a breathing mode (predator-prey type ionization instability). The observation that the amplitude of the discharge current oscillation is nearly equal to the DC value of the signal is not uncommon and has been observed in other Hall thruster setups capable of measuring the discharge current at high enough frequencies to capture this mode $\mathrm{e}^{5-7}$. The other three traces in Figure 2 demonstrate relative stability of the rest of the signals. The inner-magnet input signal is a chirp waveform written as follows, 


$$
\begin{aligned}
& y_{\text {chirp }}(t)=A \cdot \sin (2 \pi \cdot f(t) \cdot t) \\
& f(t)=f_{\text {start }}+\frac{\left(f_{\text {stop }}-f_{\text {stop }}\right)}{t_{\text {sweep }}} \cdot t \quad \text { Eqn.l }
\end{aligned}
$$

Where $f_{\text {start }}$ and $f_{\text {stop }}$ specify the range of frequencies in the sweep of duration $t_{\text {sweep }}$. Figure 2 below only shows a small section of the chirp waveform $-1 / 1250^{\text {th }}$ of the total sweep - so that the continually changing frequency is not immediately apparent.

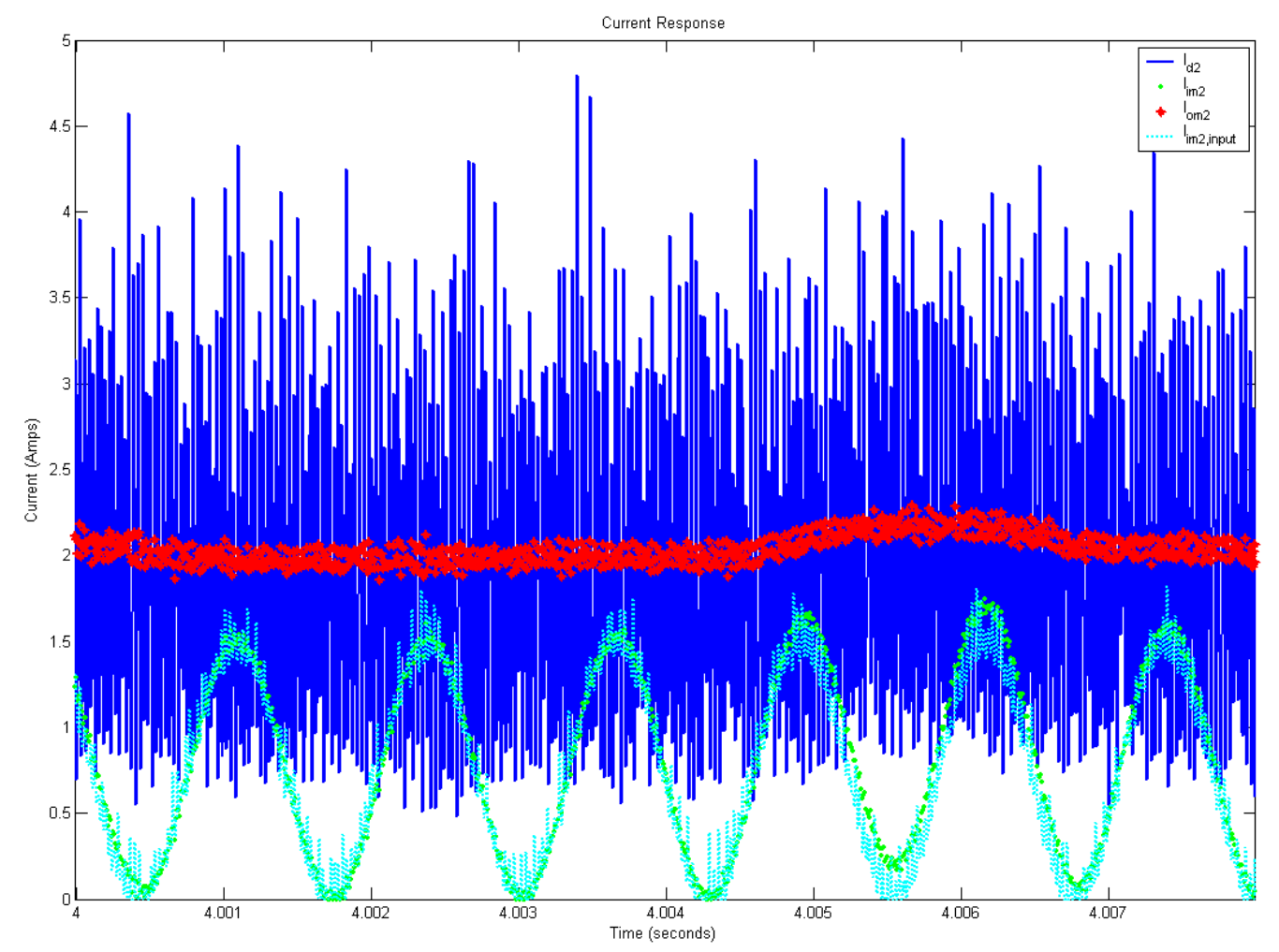

Figure 2. Time response of chirp input signal. Portion of chirp signal inner-magnet input swept from $20 \mathrm{~Hz}$ to $20 \mathrm{kHz}$ over 10 seconds.

The chirp signal is generated using a Wavetek function generator, and then sampled along with the current measurements to ensure coherence. Although one can characterize a system's dynamic response using various input signals other than chirp type waveforms, the chirp approach was used in this study because response in the time domain data would very clearly show unexpected latencies that might have been overlooked. Indeed, future experiments will utilize windowed white noise which contains a more uniform distribution of energy within the specified frequency range $f_{\text {start }}$ to $f_{\text {stop }}$ and prevents signal saturation that can easily occur with chirp experiments at unstable modes.

\section{Power Spectrum}

Performing a fast Fourier transform (FFT) on time domain data allows for the examination of the frequency content of the time data. In accordance with the Nyquist criterion, one can only measure meaningful (alias free) frequencies of half the rate of sampling. Since we sampled the time data in Figure 2 at a rate of $125 \mathrm{kHz}$ for a length of 10 seconds we can effectively determine a power spectrum for each signal from $0.05 \mathrm{~Hz}$ to $62.5 \mathrm{kHz}$. To better focus on the dynamic characteristics we could simply subtract the mean from the data, but instead we only show the frequency range from $10 \mathrm{~Hz}$ to $62.5 \mathrm{kHz}$ : 


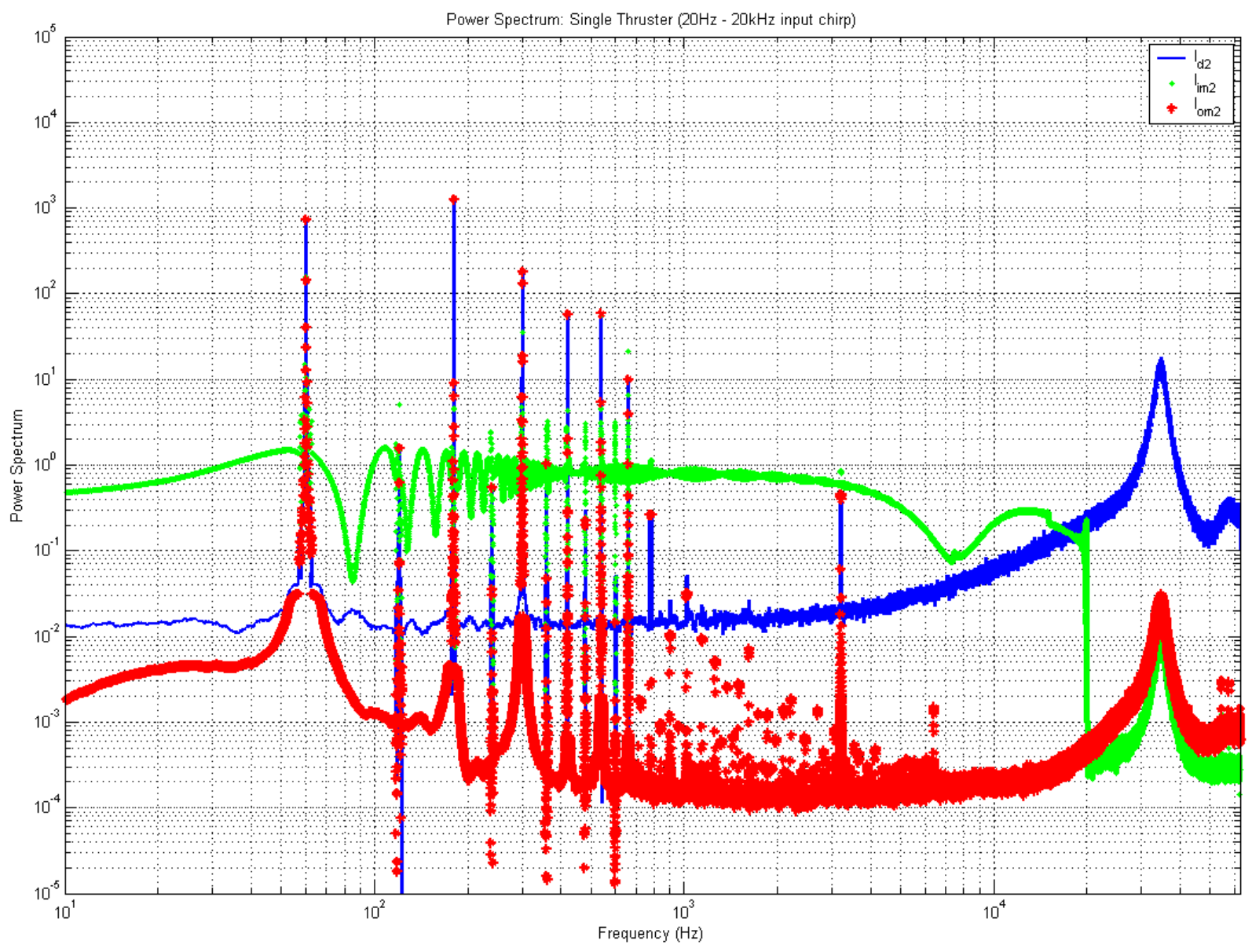

Figure 3. Power Spectrum. Single thruster operation with chirp input to $I_{i m 2}$ ranging from $20 \mathrm{~Hz}$ to $20 \mathrm{kHz}$.

We have omitted the input signal spectrum since it very closely matches the $I_{\text {om } 2}$ signal's spectrum. The two important features to notice in these data are the $60 \mathrm{~Hz}$ (power line spike, with harmonics) and the higher frequency $34.7 \mathrm{kHz}$ breathing mode. It is interesting to note that the breathing mode instability is contained in not just the discharge current data but the electromagnetic currents as well! This demonstrates that the breathing mode plasma oscillations in the discharge channel are picked up by the electromagnet circuits, implying that the coils appear to work like antennas as well as magnetic field sources. Just as antennas can receive signals they should also be able to transmit signals at this same frequency, thereby allowing for direct control of the discharge current breathing mode via the electromagnetic circuit. Our chirp input waveform gives rise to the clipped sine-like appearance of the $I_{i m 2}$ power spectrum (this is why white noise input signals are preferred). Comparing magnitudes of the signals clearly shows the effect that the $20 \mathrm{~Hz}-20 \mathrm{kHz}$ chirp has on $I_{i m 2}$ 's dynamic response as its magnitude is dramatically increased in the chirp's frequency window with a sharp drop-off at $20 \mathrm{kHz}$ as expected. The same plot without a chirp signal (i.e. a constant $I_{i m 2}$ setting) would show the power spectra of $I_{i m 2}$ and $I_{o m 2}$ nearly to be identical.

We can increase the upper and lower sweep frequencies to obtain the dynamic response of a greater range of frequencies as is shown in Figure 9. At these higher frequencies our inner-magnet power supply frequency limitations became clear. The rated frequency response of the KEPCO bipolar power supply used for the innermagnet is $<20 \mathrm{kHz}$ so the dynamic response diminishes beyond this frequency. The notch in the $I_{i m 2}$ response at about $7.5 \mathrm{kHz}$ remains unchanged with the higher frequency chirp range. This motivated an examination of the power spectrum at a lower frequency range exclusive of dynamic character at $7.5 \mathrm{kHz}$. Figure 10 shows a chirp input between $2 \mathrm{~Hz}$ to $2 \mathrm{kHz}$, and this time we do not see the $7.5 \mathrm{kHz}$ notch! The absence of this feature clearly indicates that this notch is a dynamic feature likely associated with the inductance of the inner magnetic circuit. 


\section{J. Bode Plots Analysis}

A more powerful means of analyzing dynamic signals than mere power spectra is the Bode plot which directly relates an input signal to any output signal. In this manner we can quantify to what extend our input chirp signal affects the discharge current of the thruster as well as the other discharge currents and magnetic circuit currents in clustered operation. For the power spectrum data shown in Figure 3 we produce the following Bode plot:
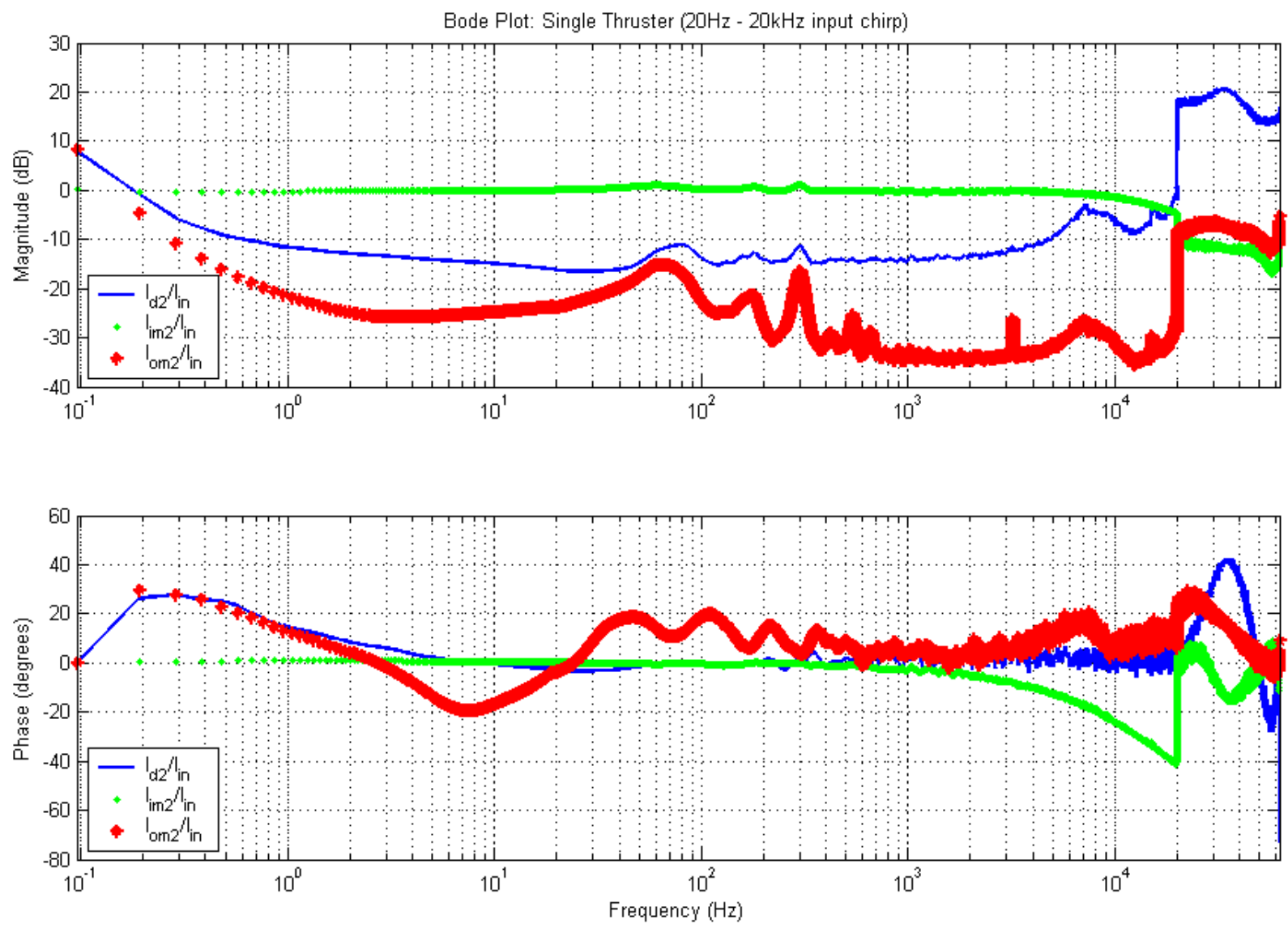

Figure 4. Bode Plot of Single Thruster, using chirp input $20 \mathrm{~Hz}-20 \mathrm{kHz}$ including amplitude and phase for all dynamic currents present in the operation of a single Hall thruster.

This perspective of the frequency response shows the 1:1 correlation observed (and expected) between the input to the inner magnet and the output of the inner magnet: $0 \mathrm{~dB}$ and flat $( \pm 5 \mathrm{~dB})$ from $\mathrm{DC}$ to $20 \mathrm{kHz}-$ this exactly how the power supply is specified to perform. The phase portion of the Bode plot (lower set of traces) shows the effective delay (relative to each frequency) between the input and output signals. Fast slew rate of the inner magnet power supply enables virtually no delay in the $I_{\text {im } 2}$ current responding to the $I_{\text {in }}$ input signal until about $20 \mathrm{kHz}$. The sharp transition in phase at $20 \mathrm{kHz}$ occurs since that is the $f_{\text {stop }}$ frequency of the input chirp; we are not inputting any energy into the system at frequencies beyond $f_{\text {stop }}$ but the data in this range is still valid (high frequency noise in the $I_{i m 2}$ signal acts as a weaker amplitude input source). Indeed, data in the frequency range $20 \mathrm{kHz}$ to $62.5 \mathrm{kHz}$ also may show how the system responds to the breathing mode (and perhaps other modes) picked up by the antenna like action of the inner electromagnetic coils.

We shall next use this Bode plot analysis to examine the effect of running the full four thruster BHT-600 cluster upon the various current signals from each of the different thrusters. A chirp input is still used to excite the $I_{\text {im } 2}$ current of thruster number 2 . The thrusters in the cluster are numbered sequentially beginning with the upper left thruster in a counter-clock-wise manner (see Figure 1). For following results we only display the signals of thruster 1 and thruster 2 though all four thrusters where in continuous operation. First, we evaluate the discharge and inner magnet currents: 

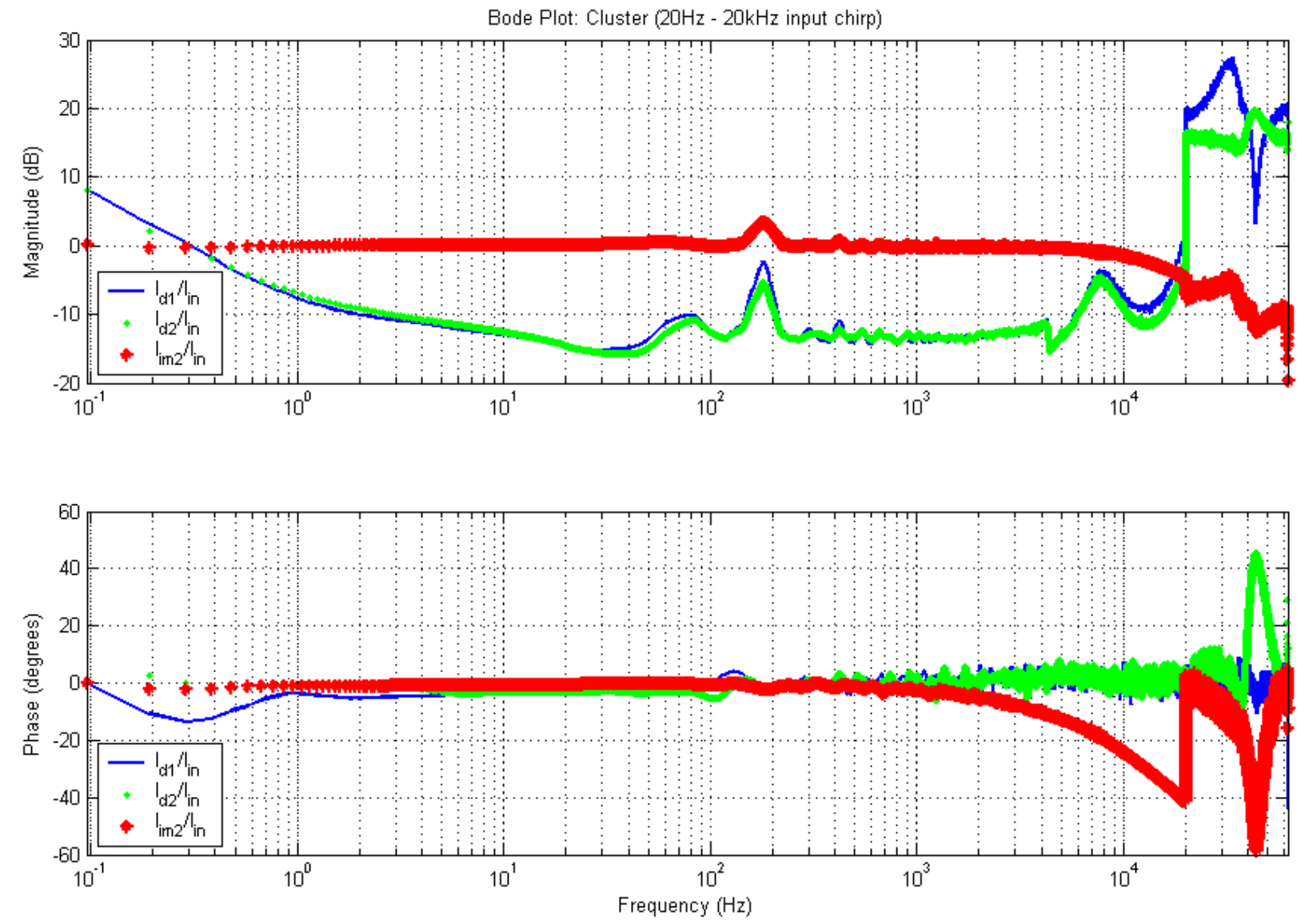

Figure 5. Bode plot for clustered operation. Chirp input provided to inner magnet of thruster 2 while a DC current of $2 \mathrm{~A}$ is provided to the other 3 inner magnets.

One of the first features of interest is the difference in breathing modes for each thruster:

Thruster 1 mode, $f_{\text {breathing }}=34.7 \mathrm{kHz}$ (constant $I_{\text {iml }}$ input)

Thruster 2 mode, $f_{\text {breathing }}=43.7 \mathrm{kHz}$ (dynamic chirp $I_{\text {im } 2}$ input)

The most likely reason for this shift is the difference in the inner magnetic current settings since the signal to thruster 1 is DC (mean value 2A) whereas the signal to thruster 2 has a lower mean DC value ( 1A) and has high dynamic AC content $(20 \mathrm{~Hz}$ to $20 \mathrm{kHz})$. Next and perhaps most intriguing, is that the magnitude of the second thruster's dynamic discharge current is lower than observed in the first thruster. While a feedback loop has not been closed at this point, we see that our chirp signal is (unexpectedly) stabilizing the discharge current oscillation in a feedforward (or open loop) manner. Overall, the signals from the different thrusters show exceptional frequency response similarity - implying low error due to manufacturing tolerances between the different thrusters in the cluster.

Next, we examine the effects clustering by comparing the Bode plots for thruster 2 when it is operated singly and during clustered operation. 

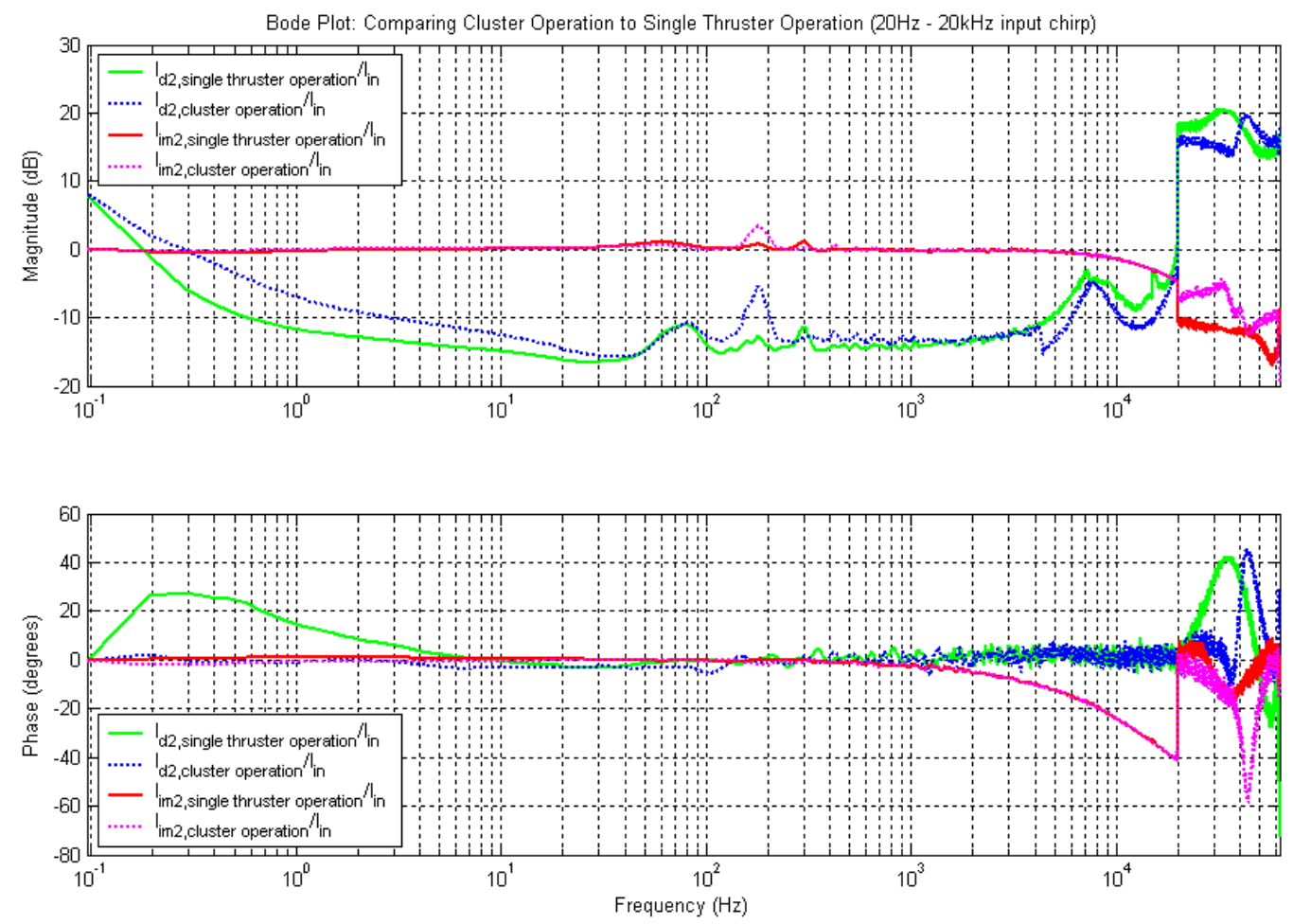

Figure 6. Effects of Clustering on Frequency Response. Both single and clustered data is taken from thruster 2 with a chirp input to the inner magnet.

Here we notice the same frequency shift in the breathing mode as observed in Figure 6Figure 5:

Thruster 2 mode, $f_{\text {breathing }}=34.7 \mathrm{kHz}$ (single thruster with dynamic chirp $I_{\text {im } 2}$ input)

Thruster 2 mode, $f_{\text {breathing }}=43.7 \mathrm{kHz}$ (clustered thrusters with dynamic chirp $I_{i m 2}$ input)

This similar shift means that the dynamic content of the $I_{i m 2}$ signal and the clustered operation are both critical in causing the breathing mode to alter its frequency. Thus, the mean DC value of the inner magnet current does not play a main role in shifting the breathing mode frequency in either single or clustered operation.

\section{K. Breathing Mode and Characteristic Length of Ionization Zone}

We are in a position to compute the characteristic length of the ionization zone that is associated with the two different breathing mode frequencies. A physical analysis the Hall thruster ionization and acceleration process ${ }^{14}$ using actual measured values from the Busek BHT-600 cluster (and the similar P5 Hall thruster, NASA-173Mv1 ${ }^{23}$ ) along with a simple energy balance produces the following empirical scaling for the BHT-600 thrusters:

$$
L_{\text {char., ioniz. }}=\frac{9997}{f_{\text {breathing }}} \approx \frac{10000}{f_{\text {breathing }}} \quad \text { Eqn.2 }
$$

Using this scaling, the following characteristic ionization lengths for the different breathing modes are:

\begin{tabular}{lll}
$\boldsymbol{f}_{\text {breathing }}$ & $\boldsymbol{L}_{\text {char, }, \text { ioniz. }}$ & Conditions $\boldsymbol{f}_{\text {breathing }}$ is observed \\
\hline $34.7 \mathrm{kHz}$ & $29 \mathrm{~cm}$ & Non-dynamic $\boldsymbol{I}_{\text {im }}$ or Non-Clustered \\
$43.7 \mathrm{kHz}$ & $23 \mathrm{~cm}$ & Dynamic $I_{\text {im }}$ and Clustered
\end{tabular}

Table 1. Breathing mode length scaling 
A $26 \%$ decrease in the relative length of the ionization zone is observed for the clustered cases with dynamic (chirp) input to the inner magnet. This indicates that a compression of the ionization zone occurs as a result of both plume interaction along with dynamic electromagnet control. Plume interactions have been observed to give rise to a focusing effect ${ }^{5}$ which would naturally involve a compression of the overall plume thereby reducing the characteristic ionization length. Faraday's law,

$$
\nabla \times E=-\frac{\partial B}{\partial t} \quad E q n .3
$$

explicitly shows that an unsteady radial magnetic field - like the dynamic chirp input (Eqn. 1) - can give rise to perpendicular (azimuthal and axial) electric fields, thus providing direct interaction with ions inside and near the exit plane of the discharge channel. Additional acceleration of the ions could certainly lead to a shift in the breathing mode frequency since it is an instability that is driven by continuity of heavy particles.

\section{Preliminary Closed Loop Feedback Control Results}

For the following tests we ran only thruster number 2 and implemented a control loop like the one shown in Figure 1. While feedback control is theoretically possible with our setup at rates as high as $100 \mathrm{kHz}$, the following tests where each run at $20 \mathrm{kHz}$. A basic lead-lag controller is used to study the basic effectiveness of feedback control:

$$
H_{\text {controller }}=\frac{k_{d} z^{2}+k_{p} z+k_{i}}{z} \quad \text { Eqn. } 4
$$

Over thirty different gain settings were tested with various levels of success; we present two such cases next:
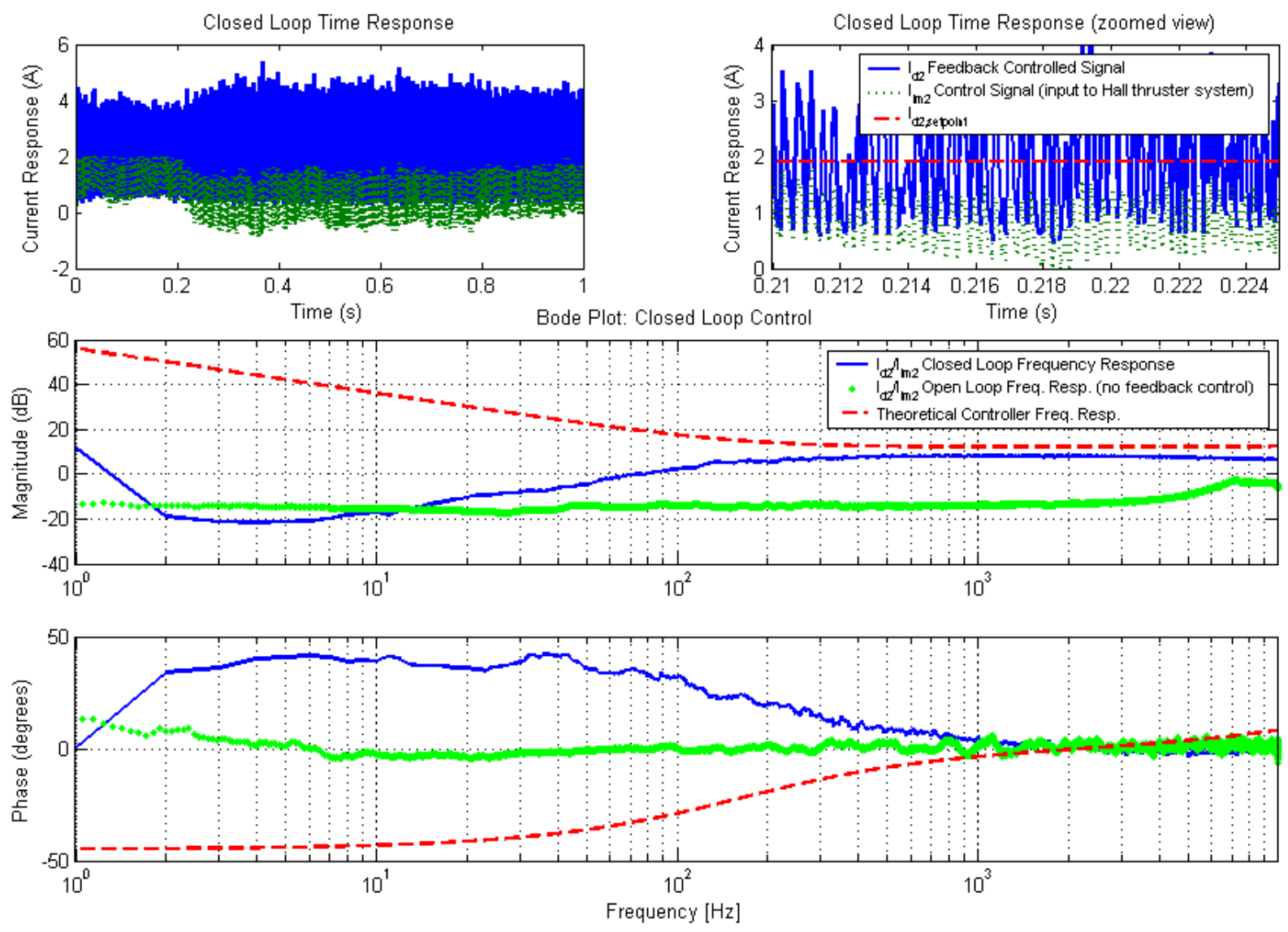

Figure 7. Stabilized $\boldsymbol{I}_{d 2}$ response. Here we have some degree of stabilization in the elimination of the small mode at approximately $7 \mathrm{kHz}$ (comparing open and closed loop responses). 

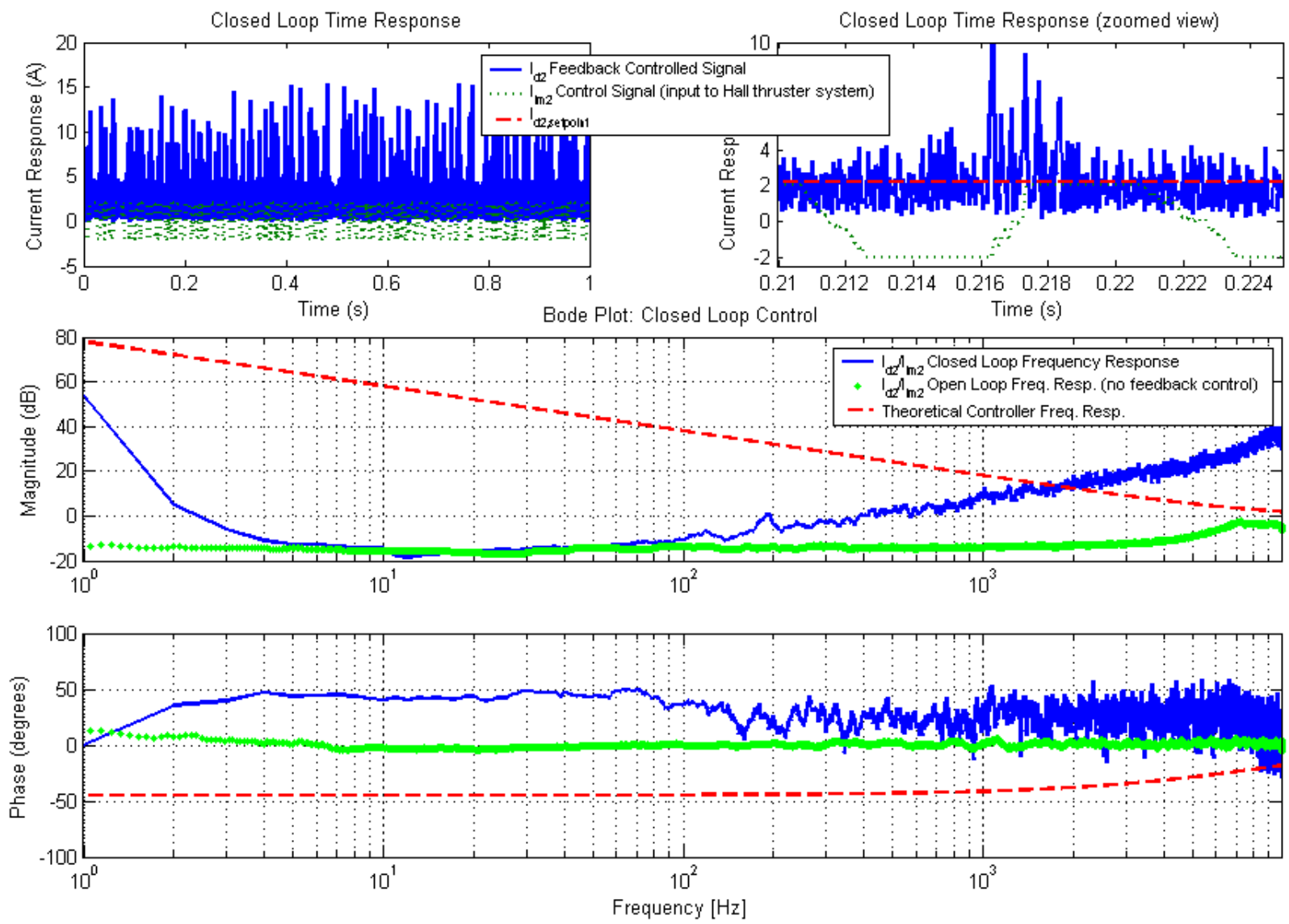

Figure 8. De-stabilized $\boldsymbol{I}_{d 2}$ response! Here we have entirely destabilized the thruster by poor choice of our gain parameters. The magnitude of this de-stabilization illustrated vividly by comparing our open loop discharge current oscillations which peaked at about 4.5A (Figure 2) to the above discharge oscillations which peak as high as 15A!

The gains used in our controller for the data shown in Figure 7 are: proportional gain, $k_{d}=4$, integral gain, $k_{i}=$ 4000 , and differential gain, $k_{d}=0.00002$. Flatting of the mode at $7 \mathrm{kHz}$ shows some effectiveness of this controller. The gains used in our controller for the data shown in Figure 8 are: $k_{d}=1, k_{i}=50000$, and $k_{d}=0.000001$. This later case is clearly unstable as can be seen in the amplification of the $7 \mathrm{kHz}$ mode. The slower sample rate $(20 \mathrm{kHz}) \mathrm{in}$ the feedback controllers tested does not enable direct control the breathing modes ( $35 \mathrm{kHz}$ to $44 \mathrm{kHz}$ ) so future work will approach this more challenging problem. Careful use of system identification data (i.e. the open loop response Bode plots) and cleverly designed - more sophisticated (higher order) - controllers should enable successful feedback control of the breathing modes and other Hall thruster instabilities.

\section{Conclusion}

A custom high speed $(100 \mathrm{kHz}$ to $500 \mathrm{kHz})$ DAQ control system was used to evaluate the dynamics of Hall thruster operation. Time domain data was acquired and converted to frequency space in the form of power spectrum and Bode plots. The effects of clustering and the effects of a dynamic inner magnet chirp input were examined and the breathing mode was observed to increase from $34.7 \mathrm{kHz}$ to $43.7 \mathrm{kHz}$ when excited in the clustered state. An ionization zone length scaling was computed for the BHT-600 for both single and clustered operation. Finally, a feedback control loop was closed at $20 \mathrm{kHz}$ and direct control of the discharge current oscillations was demonstrated using the inner magnet current as the control signal. 


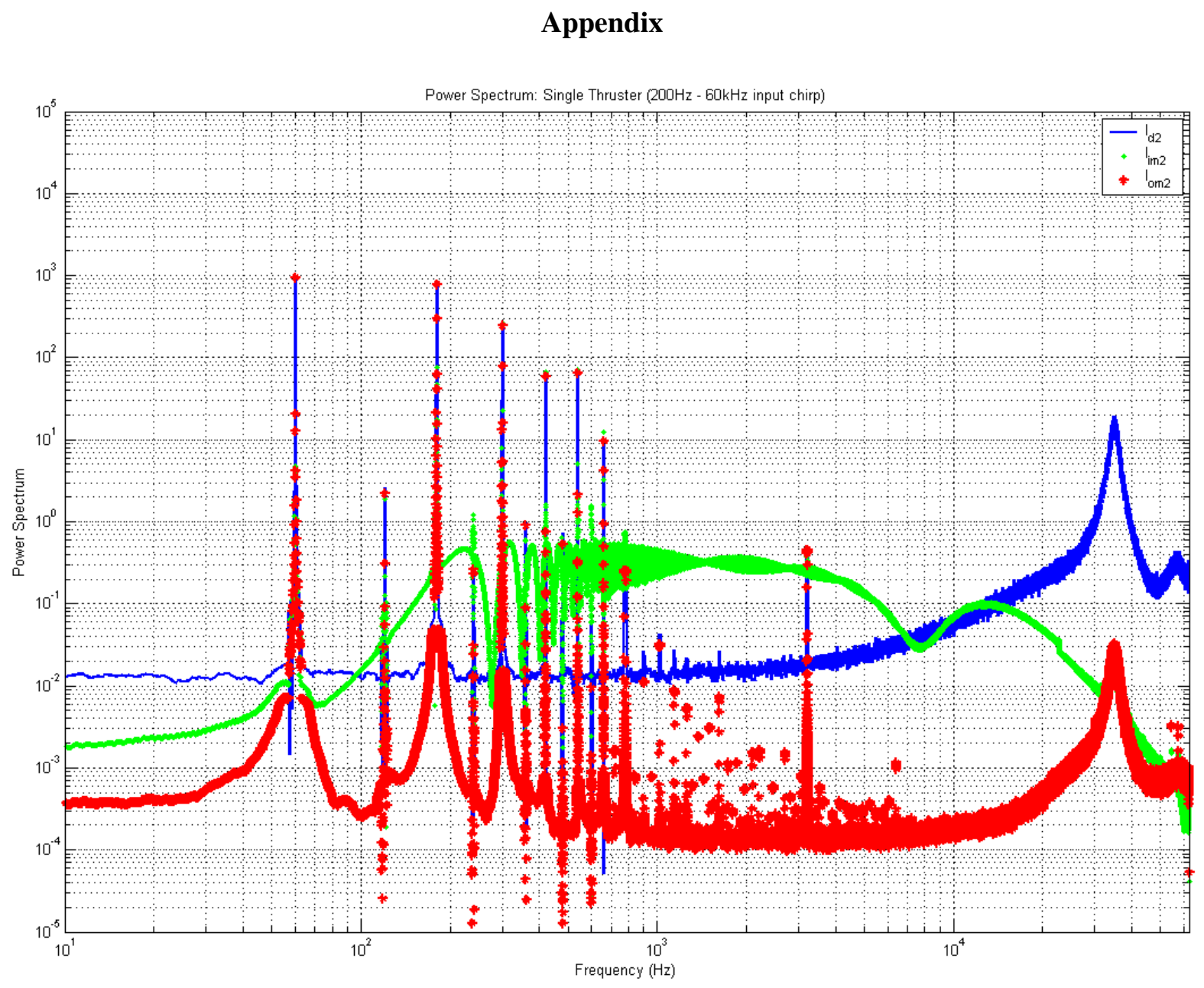

Figure 9. Power Spectrum Response of Higher Chirp Frequencies. Single thruster operation with chirp input to $I_{i m 2}$ ranging from $200 \mathrm{~Hz}$ to $60 \mathrm{kHz}$. 


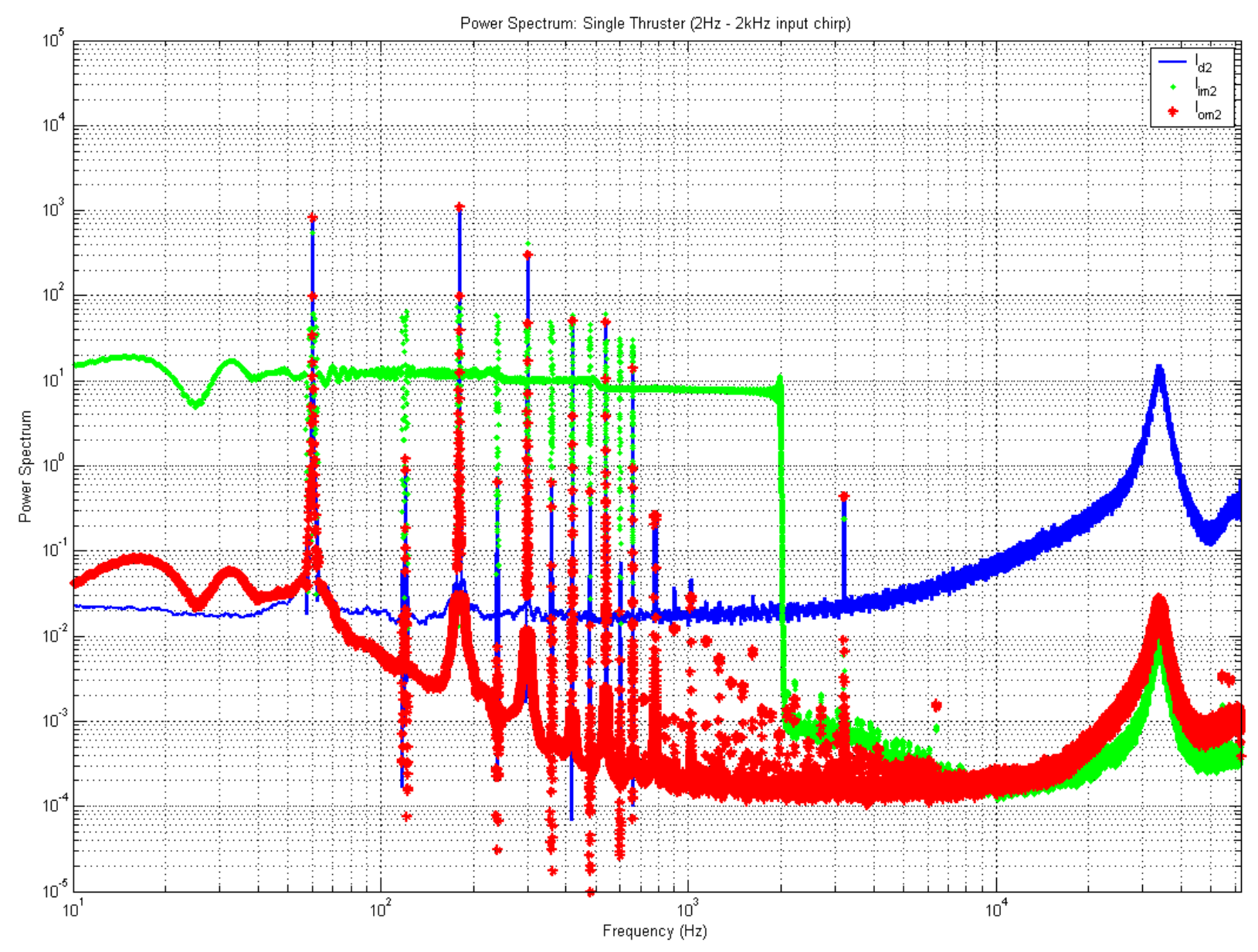

Figure 10. Power Spectrum Response of Lower Chirp Frequencies. Single thruster operation with chirp input to $I_{i m 2}$ ranging from $2 \mathrm{~Hz}$ to $2 \mathrm{kHz}$.

\section{Acknowledgments}

The Author thanks fellow graduate students, Jesse Linnell and Justin ("kooj”) Koo for their many discussions as well as Busek V.P., Bruce Pote for his technical aid in the preliminary operation of the BHT-600 cluster in the PEPL Large Vacuum Test Facility.

\section{References}

${ }^{1}$ Spores, R.A., Spanjers, G.G., Birkan, M., Lawrence, T.J., "Overview of the USAF Electric Propulsion Research Program," AIAA-2001-3225, 37th AIAA Joint Propulsion Conference, July 2001, Salt Lake City, UT.

${ }^{2}$ Dunning, J.W., Benson, S., Oleson, S., "NASA's Electric Propulsion Program," IEPC-01-002, 27th International Electric Propulsion Conference, October 2001, Pasadena, CA.

${ }^{3}$ Williams, L.A., Engel, M., and Dolgopolov, A., "Commercial Satellite and Launch Vehicle Buying Trends," AIAA-2002980, 20th AIAA International Communications Satellite Conference and Exhibit, May 2002, Montreal, Quebec.

${ }^{4}$ Hargus, W.A, Reed, G., "The Air Force Clustered Hall Thruster Program," AIAA-2002-3678, $38^{\text {th }}$ AIAA Joint Propulsion Conference and Exhibit, July 2002, Indianapolis, IN.

${ }^{5}$ Beal, B.E., "Clustering of Hall Effect Thrusters for High-Power Electric Propulsion Applications," Ph.D. Dissertation, University of Michigan, 2004.

${ }^{6}$ Hargus, W.A., Pote, B., "Examination of a Hall Thruster Start Transient," AIAA-2002-3956, 38 ${ }^{\text {th }}$ AIAA Joint Propulsion Conference and Exhibit, July 2002, Indianapolis, IN.

${ }^{7}$ Hargus, W. A., Meezan, N.B., Cappelli, M.A., "The Transient Behavior of a Low Power Laboratory Xenon Hall Thruster," AIAA-97-3050, 33 ${ }^{\text {rd }}$ AIAA Joint Propulsion Conference and Exhibit, July 1997.

${ }^{8}$ Chesta, E., Lam, C. M., Meezan, N.B., Schmidt, D. P., Cappelli, M. A., “A Characterization of Plasma Fluctuations within a Hall Discharge," IEEE Transactions on Plasma Science, Vol. 29, No. 4, August 2001. 
${ }^{9}$ Chesta, E., Meezan, B., Cappelli, M.A., "Stability of a magnetized Hall plasma discharge," Journal of Applied Physics, Vol. 89, No. 6, March 2001.

${ }^{10}$ Choueiri, E.Y., "Plasma oscillations in Hall thrusters," Physics of Plasmas, Vol. 8, No. 4, April 2001.

${ }^{11}$ Gascon, N., Perot, C., Bonhomme, G., Caron, X., Bechu, S., Lasgorceix, P., Izrar, B., Dudeck, M. "Signal Processing and Non-Linear Behavior of a Stationary Plasma Thruster: First Results," AIAA-99-2427, 35th AIAA Joint Propulsion Conference and Exhibit, July 2000, Los Angeles, CA.

${ }^{12}$ Choueiri, E.Y., "Characterization of Oscillations in Closed Drift Thrusters," AIAA-94-3013, 30th AIAA Joint Propulsion Conference and Exhibit, July 1994.

${ }^{13}$ Baranov, V.I., Nazarenko, Y.S., Petrosov, V.A., Vasin, A.I., Yashnov, Y.M., "Theory of oscillations and conductivity for Hall thruster," AIAA-96-3192, $32^{\text {nd }}$ AIAA Joint Propulsion Conference and Exhibit, July 1996, Buena Vista, FL.

${ }^{14}$ Fife, J.M., Martinez-Sanchez, M., Szabo, J., "A Numerical Study of Low-Frequency Discharge Oscillations in Hall Thrusters," AIAA-97-3052, 33 ${ }^{\text {rd }}$ AIAA Joint Propulsion Conference and Exhibit, July 1997.

${ }^{15}$ Litvak, A. A., Raitses, Y., Fisch, N. J., "Experimental studies of high frequency azimuthal waves in Hall thrusters," Physics of Plasmas, Vol. 11, No. 4, April 2004.

${ }^{16}$ Yamamoto, N,, Komurasaki, K., Arakawa, Y., “A Suppression method of Discharge Oscillations in a Hall Thruster,” AIAA2004-3678, 40 $0^{\text {th }}$ AIAA Joint Propulsion Conference and Exhibit, July 2004, Fort Lauderdale, FL.

${ }^{17}$ Raitses, Y., Fisch, N. J., Ertmer, K. M., Burlingame, C. A., "A Study of Cylindrical Hall Thruster for Low Power Space Applications," AIAA-2000-3421, 36th AIAA Joint Propulsion Conference and Exhibit, July 2000.

${ }^{18}$ Chu, T.K., Hendel H.W., Feedback and Dynamic Control of Plasma, American Institute of Physics Conference Proceedings, 1970.

${ }^{19}$ Okabayashi, Bialek, Chance, Chu, Fredrickson, Gorafalo, Gryaznevich, Hatcher, Jensen, Johnson, La Haye, Lazarus, Makowski, Manickam, Navratil, Scoville, Strait, Turnbill, Walker, and the DIII-D Team, "Active feedback stabilization of the resistive wall mode on the DIII-D device," Physics of Plasmas, Vol. 8, No. 5, May 2001.

${ }^{20}$ Koo, J., Keidar, M., Boyd, I., "Boundary Conditions for a 2-D Hybrid Stationary Plasma Thruster Model," AIAA-20043781, 40 th AIAA Joint Propulsion Conference and Exhibit, July 2004, Fort Lauderdale, FL.

${ }^{21}$ Boozer, A.H., "Feedback equations for the wall modes of a rotating plasma," Physics of Plasmas, Vol. 6, No. 8, August 1999.

${ }^{22}$ Walker, M., Gallimore, A. D., Cai, C., Boyd, I. D., "Pressure Map of a Facility as a Function of Flow Rates to Study Facility Effects," AIAA-2002-3815, $38^{\text {th }}$ AIAA Joint Propulsion Conference and Exhibit, July 2002, Indianapolis, IN.

${ }^{23}$ Linnell, J. A., Gallimore, A. D., "Efficiency Analysis of a Hall Thruster Operating with Krypton and Xenon," AIAA-20053683, 41 st AIAA Joint Propulsion Conference and Exhibit, Tucson, Arizona, July 10-13, 2005. 